\title{
On rotational pre-alignment for tree log identification using methods inspired by fingerprint and iris recognition
}

\author{
Rudolf Schraml $^{1}(\mathbb{D}) \cdot$ Heinz Hofbauer ${ }^{1}$. \\ Alexander Petutschnigg ${ }^{2}$. Andreas Uhl ${ }^{1}$
}

Received: 30 November 2015 / Revised: 28 September 2016 / Accepted: 30 September 2016 / Published online: 4 November 2016

(C) The Author(s) 2016. This article is published with open access at Springerlink.com

\begin{abstract}
Tree log end biometrics is an approach to track logs from forest to further processing companies by means of log end images. The aim of this work is to investigate how to deal with the unrestricted rotational range of cross sections in $\log$ end images. Thus, the applicability of three different rotational pre-alignment strategies in the registration procedure is assessed. Template computation and matching is based on fingerprint and iris recognition techniques which were adopted and extended to work with log end images. To address these questions, a testset built up on 279 tree $\operatorname{logs}$ is utilized in the experiments. The evaluation assesses the basic performance of the rotational pre-alignment strategies and their impact on the verification and identification performances for different fingerprint- and iris-based configurations. Results indicate that rotational pre-alignment in the registration procedure is the main component to deal with rotation in log end biometrics. The best configurations achieve identification rates $>93 \%$. By showing that cross sections in log end images can be rotated to a distinctive position, this work is a first step towards real word log end biometrics.
\end{abstract}

Keywords Rotation invariant log end biometrics · Tree log tracking · Wood traceability - European timber regulation . Chain of custody

This work is partially funded by the Austrian Science Fund (FWF) under Project No. TRP-254.

\footnotetext{
Rudolf Schraml

rudi.schraml@gmail.com

1 Department of Computer Sciences, University of Salzburg, Salzburg, Austria

2 Department of Forest Products Technology and Timber Construction, University of Applied Sciences Salzburg, Salzburg, Austria
}

$\begin{array}{ll}\text { Abbreviations } \\ \text { CS } & \text { Cross section } \\ \text { TS } & \text { Testset } \\ \text { NORC } & \text { No rotation compensation } \\ \text { CM } & \text { Center of mass } \\ \text { EER } & \text { Equal error rate } \\ \text { MS } & \text { Matching score }\end{array}$

\section{Introduction}

Tracking of tree logs is an economic requirement to map the ownership of each log. Additionally, social aspects have become more important and sustainability certificates, e.g. Forest Stewardship Council (FSC), are a must have for all end-sellers. Finally, traceability is legally mandated by the European Timber Regulation or the U.S. Lacey Act to prohibit illegal logging.

In the industry, commonly approaches which rely on physically marking each log are utilized and radio-frequency identification (RFID) is promoted as future technology. Without doubt RFID technology shows a lot of advantages. However, physical marking requires time and suffers costs. Wood log biometrics is a physically marking-free approach to establish log traceability.

By superficially comparing annual ring patterns of log ends to the patterns of human fingerprints, one finds a close resemblance. Wood logs offer characteristics on log end faces in terms of annual rings, pith position, shape and dimension. The pith as growth centre is an unique reference point which is similar to the core point of a fingerprint. Furthermore, $\log$ ends are circularly shaped and in combination with the pith the annual ring pattern can be polar-transformed like the texture of an iris. 
Our research on log end biometrics [13-16] treated fundamental questions related to biometric tracking of wood logs using fingerprint and iris recognition based approaches. In Ref. $[14,16]$ we investigated the robustness of log end biometrics to temporal, longitudinal and surface variations based on cross-sectional images (CS-Images) captured in a controlled environment. In Ref. $[15,16]$ we investigated whether $\log$ end biometrics is basically suited to discriminate between 150 different logs. In Ref. [16] we assessed the discriminative power of geometric log end features and we proved their reliability in case of automated cross-sectional (CS) segmentation [17] and pith estimation [18]. Similar as in [1] the experimental evaluation showed that Zernike polynomials are the most reliable geometric features. However, the achieved verification performances showed that just geometric features are not discriminative enough for log end identification. Finally, in [15] we explored the general applicability of methods inspired by fingerprint and iris recognition to identify tree logs. Our experiments for 150 tree logs from two testsets showed that for both approaches an identification performance of $100 \%$ can be achieved.

However, so far all CS-Images of each log were captured with nearly no variation with respect to scale and rotation. Rotational variations are a key issue in log end biometrics and it is not clear if log ends can be rotated to a distinctive position just using features which can be detected in CS-Images. Furthermore, the issue of finding a distinctive rotational log position is becoming important for the sawmill industry. Research showed that log positioning at the saw intake and individual cutting increases the value of each log up to $21 \%$ [2].

In this study we assess the suitability of three different rotational pre-alignment strategies which basically rely on the pith position and the CS boundary. Suitability is therefore assessed in two steps. In the first step the basic performance of each strategy to align each CS to a distinctive position is assessed. Therefore, the results of an image registration algorithm are evaluated. In the second step, the impact of rotational pre-alignment on the verification and identification performances for a set of fingerprint- and iris-based approaches is assessed. In addition to the two testsets from [15], a third testset with 109 different tree logs, showing strong rotational variations, is utilized for the experiments.

Section 2 introduces the computation and matching of log templates for the fingerprint- and iris-based approaches. Subsequently, the experimental evaluation is presented in Sect. 3 followed by the conclusions in Sect. 4 .

\section{CS-Code computation and matching}

An exemplary enrolment and identification scheme for log end biometrics is depicted in Fig. 1. Enrolment of a tree

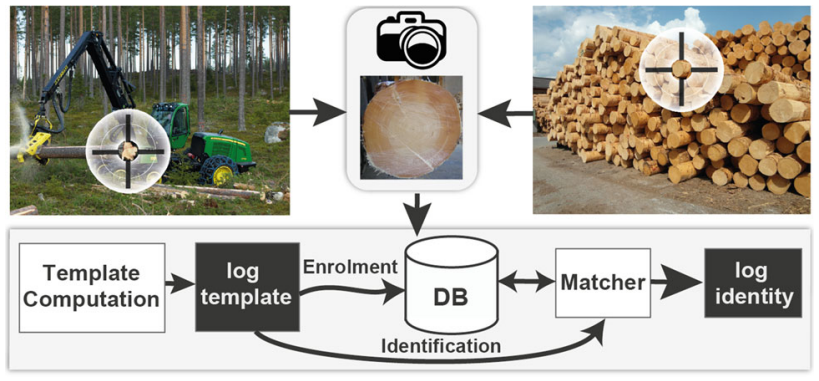

Fig. 1 Enrolment and identification schemes

$\log$ is performed in the forest. After a tree $\log$ is cut and processed by a harvester, the log end is captured by a digital camera mounted on the harvester head. Templates of logs which are computed by means of CS-Images are denoted as CS-Codes. For enrolment the computed CS-Code is stored in the database. Identification can be performed at each stage of the $\log$ processing chain where an appropriate capturing device is available. Typically, identification is required when a log is delivered to a sawmill. The CS-Image of a log, for which identification is required, is processed by the biometric system and the computed CS-Code is matched to all enrolled CS-Codes in the database. The best ranked match, if it exceeds a certain threshold, determines the identity of the $\log$.

\subsection{Rotational pre-alignment strategies}

Biometric systems compute a compact representation (=template) of a biometric characteristic captured of an individual/ object. Because of privacy, timing and memory considerations captured raw data is not stored in the system. Templates are designed in order to enable fast matching with respect to the spatial interrelationship of the extracted features. This is a key to achieve a high discriminative power, and thus registration is an important task in biometrics. Pre-alignment in the registration procedure refers to absolute alignment: The characteristic is pre-aligned independent of a reference just by using intrinsic features [8]. Image registration algorithms (see Sect. 3.2.1) require relative alignment and are not applicable in this case since a template cannot be used in a registration procedure (where two images are required). The goal of registration is to align the captured characteristic to a distinctive position independent of affine transformations like rotation or scale.

In fingerprint recognition different approaches for rotational pre-alignment were proposed. Several algorithms use the core point and a further feature (e.g. computed from the shape or the orientation field) for pre-alignment. No final solution has been found so far [8]. However, fingerprints show key points denoted as minutia and minutiae point clouds are well suited for relative alignment (e.g. [11]) in the match- 
Fig. 2 Different rotational pre-alignment strategies for CS registration are assessed

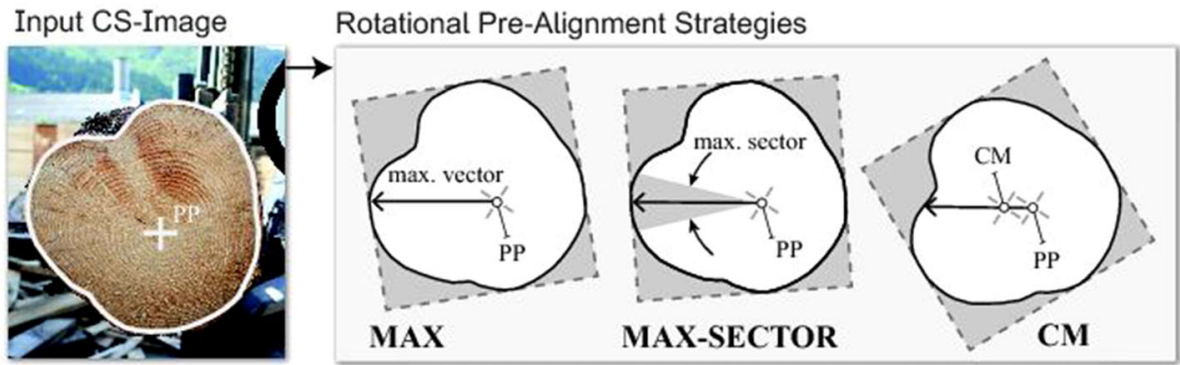

ing procedure. In case of iris recognition just small rotational variances are expected which are commonly compensated in the template matching procedure.

In $\log$ end biometrics rotation is one of the main issues. Compared to an iris, the rotational range of a log end is not restricted and compared to a fingerprint just the pith as keypoint is available for alignment. Rotation in log end biometrics needs to be considered in both, the template computation and the template matching procedure which refers to rotational pre-alignment and rotation compensation, respectively:

Rotational pre-alignment In the registration step of the template computation procedure the goal is to rotationally pre-align the CS in a log end image to a distinctive position. Optimally, CSs in different CS-Images of the same log end can be rotated to almost the same position.

Rotation compensation To compensate the remaining rotational variances different strategies in the feature extraction and template matching procedure can be applied. However, rotation compensation during feature extraction and template matching increases the computational expense and probably decreases the discriminative power.

In principle, the better CSs can be pre-aligned to distinctive positions in the registration procedure, the less other components of the biometric system have to deal with issues caused by rotational variances.

As there is no evidence whether log ends can be rotated to a unique position, we evaluate three different rotational prealignment strategies in the registration procedure to address this question. All approaches require the pith position and the CS boundary as input. Automated approaches for pith estimation and CS segmentation were presented in $[10,17]$ and [18], respectively. For each approach the rotational prealignment vector between the pith position and the CS border is determined differently (Fig. 2):

- MAX = the max. pith to border distance vector.

- MAX-SECTOR = the centre vector of the sector (angular width $=30^{\circ}$ ) containing the max. sum of the pith to border distances.

- $\mathbf{C M}=$ the pith to CS boundary vector which passes through the center of mass (CM) of the CS boundary polygon.
Subsequently, the CS is rotated around the pith in order to situate the rotational pre-alignment vector at a certain position. For this work the vector is placed on the left horizontal axis. Remaining rotational variances are considered differently for the fingerprint-based and the iris-based approaches as described in Sects.2.2 and 2.3, respectively.

\subsection{Fingerprint-based CS-Codes}

Human fingerprint recognition is well-investigated, and there exist mainly three groups of approaches: minutiae-based, correlation-based and feature-based approaches [8]. Apart from the presence of the pith as detectable feature, CS patterns do not exhibit further constant features like minutia's in fingerprints. Hence, minutiae-based approaches are not qualified for $\log$ CSs.

For this reason and same as in [13-15] the texture-featurebased approach by [4] is adopted and extended to compute and compare CS-Codes from CS-Images. Compared to the almost constant ridge frequency of a fingerprint annual ring frequencies vary significantly. Thus, the Gabor filterbank is extended to six different filters instead of a single one. For preprocessed CS-Images scaled to 512 pixels we suggest the following Gabor filterbank settings to capture the expected variety of occurring annual ring widths: $G(\lambda, \theta, \sigma, \gamma)=$ $G(\lambda, \sigma)=((2,1),(3.5,2),(4.5,3),(5.5,3),(6.5,3),(7.5,3)), \theta=$ $\{0,22.5, \ldots, 135,157.5\}, \gamma=0.7 . \lambda$ is the filter wavelength, $\theta$ represents the orientation, $\sigma$ is the standard deviation and $\gamma$ specifies the filter aspect ratio.

Remaining rotational variances are compensated by repeatedly computing features for rotated versions of the already rotation compensated CS-Image. All feature vectors computed for different rotations $\left(\Theta_{1}, \ldots, \Theta_{n}\right)$ compose the CS-Code of a CS-Image. The amount of feature vectors computed for different rotations has to be chosen carefully, because each additional feature vector suffers computational expense.

The computation of a feature vector $\left(\Theta_{i}\right)$ for a certain rotation is performed in four steps (see Fig. 3). As input the CS-Image, pith position, CS boundary and the rotational prealignment vector $\Theta_{0}$ are required. First, and according to the rotation of $\Theta_{i}$ the CS-Image is rotated around the pith and 
Fig. 3 Fingerprint-based template computation: Feature vectors are computed in a predefined sector specified by the rotational pre-alignment vector $\Theta_{0}$

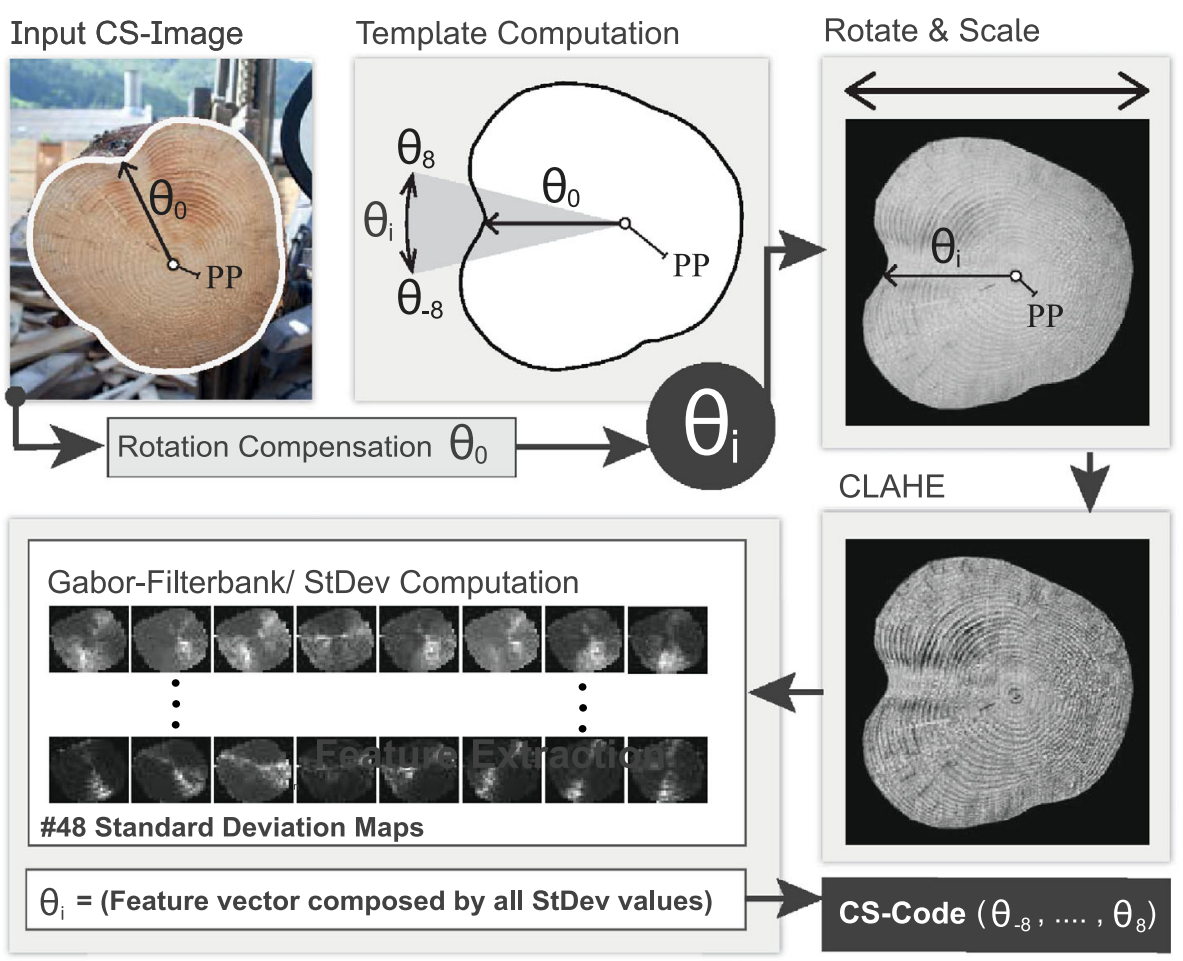

the CS is cropped to the CS boundary box and scaled to 512 pixels in width. Subsequently, the rotated, cropped and scaled CS-Image is enhanced. Commonly, the annual ring pattern is disturbed due to cutting and there arise different types of intraclass CS variations in real-world identification scenarios [13]. The purpose of enhancement is to strengthen the annual ring pattern contrast and to compensate CS variations. In this work for enhancement contrast limited adaptive histogram equalization (CLAHE, [6]) is applied to the registered image.

The enhanced CS-Image is filtered with each filter in the filterbank. The filtered images are further subdivided into blocks ( $16 \times 16$ pixels). For all blocks of each filtered image, the grey value standard deviations are computed and stored into a matrix. Values of blocks which are not within the CS border are assigned with a marker value. These markers are used to discriminate between background and CS in the matching procedure. All matrices are stored as onedimensional vector.

Fingerprint-based matching procedures Matching between two CS-Images is performed by computing the minimum matching score $(\mathrm{MS})$ between all feature vectors $\left(\Theta_{1}, \ldots, \Theta_{n}\right)$ of the CS-Codes from both CS-Images.

Like in Ref. [13] three different matching procedures are evaluated to investigate the discriminative power of the annual ring pattern, the shape and a fusion of both: The first procedure which just considers annual ring pattern information is denoted as annual ring pattern $\mathrm{MS}\left(\mathrm{MS}_{\mathrm{AP}}\right)$ and is defined as the minimum matching score between the feature vectors of both CS-Codes:

$$
\begin{array}{r}
\text { MS }{ }_{A P}\left(\mathrm{CS}-\mathrm{Code}_{1}, \mathrm{CS}-\mathrm{Code}_{2}\right)=\min \mathrm{MS}\left(\theta_{i}, \theta_{j}\right) \\
\text { where } \theta_{i} \in \mathrm{CS}-\operatorname{Code}_{1}\left(\theta_{0}, \ldots, \theta_{358}\right), \\
\theta_{j} \in \mathrm{CS}-\operatorname{Code}_{2}\left(\theta_{0}, \ldots, \theta_{358}\right)
\end{array}
$$

Due to interpolation in the registration procedure (rotation and scaling) the best MS is achieved when comparing all feature vectors of both CS-Codes. The MS between two feature vectors of two CS-Codes is computed by:

$\operatorname{MS}\left(\theta_{i}, \theta_{j}\right)=\frac{1}{M} \sum_{k=0}^{n} D\left(\theta_{i}(k), \theta_{j}(k)\right)$

where $\theta_{i}, \theta_{j}$ are two feature vectors of the CS-Codes which are compared, $k$ specifies the index of the feature value in both vectors, $n$ is the max. number of feature values and $M$ is a normalization factor. As distance function $(D)$ the L1 norm is utilized (as our experiments showed that the L1 norm performs better than the L2 norm [14]):

$D= \begin{cases}\left|\theta_{i}(k)-\theta_{j}(k)\right| & \text { if } k \in M C S_{i} \cap M C S_{j} \\ 0 & \text { otherwise }\end{cases}$

As noted, background feature values are specified by a certain marker. Hence, we define $\mathrm{MCS}_{i}$ and $\mathrm{MCS}_{j}$ as the corresponding masks of the feature vectors which allow to differentiate between background and CS. Just feature vector value pairs which are in the intersection of both CSs are utilized for computing $\mathrm{MS}_{\mathrm{AP}}$ and the score is normal- 
Fig. 4 Iris-based template computation: CS-Images are polar-transformed and enhanced using CLAHE

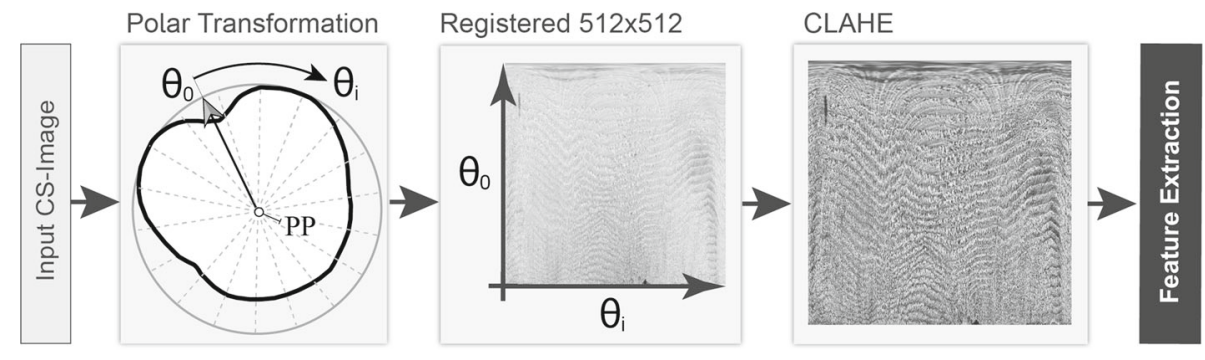

ized by the amount of the considered feature value pairs: $M=\left|\mathrm{MCS}_{1} \cap \mathrm{MCS}_{2}\right|$.

The second procedure $\left(\mathrm{MS}_{F}\right)$ is a measure describing the similarity of the shapes of two CSs. $\mathrm{MS}_{F}$ is defined as the minimum False Negative Mask Overlap Score $(F)$ between the masks of the feature vectors of both CS-Codes:

$\mathrm{MS}_{F}\left(\mathrm{CS}-\mathrm{Code}_{1}, \mathrm{CS}-\mathrm{Code}_{2}\right)=\min F\left(\mathrm{MCS}_{i}, \mathrm{MCS}_{j}\right)$

The false negative mask overlap score $(F)$ between two different masks $\left(\mathrm{MCS}_{i}, \mathrm{MCS}_{j}\right)$ is defined as the ratio between the symmetric difference $(\triangle)$ from both masks and the area of the smaller mask:

$F\left(\mathrm{MCS}_{i}, \mathrm{MCS}_{j}\right)=\frac{\left|\mathrm{MCS}_{i} \triangle \mathrm{MCS}_{j}\right|}{\min \left(\left|\mathrm{MCS}_{i}\right|,\left|\mathrm{MCS}_{j}\right|\right)}$

Finally, the last procedure $\left(\mathrm{MS}_{\mathrm{AP}, F}\right)$ is based on score level fusion [5, p.225]. Therefore, the $\mathrm{MS}_{\mathrm{AP}}$ and $\mathrm{MS}_{F}$ scores are mapped to the $[0,1]$ range using min-max normalization. The lower and upper bounds of the score ranges are determined using the scores computed in the experiments. For score level fusion the normalized scores $\mathrm{MS}_{\mathrm{AP}}^{\prime}$ and $\mathrm{MS}_{F}^{\prime}$ are utilized:

$\mathrm{MS}_{\mathrm{AP}, F}=\frac{\mathrm{MS}_{\mathrm{AP}}^{\prime}+M S_{F}^{\prime}}{2}$

\subsection{Iris-based CS-Codes}

For the iris-based approaches the pith position is used as reference point to polar transform the CS-Image using bi-cubic interpolation. The registration compensation vector $\left(\Theta_{0}\right)$ computed by one of the rotational pre-alignment strategies is therefore used as initial vector for the polar transformation. $\Theta_{0}$ is aligned at the left boundary of the polar-transformed CS-Image (see Fig. 4). For normalization each pixel in the polar image is stretched according to the length of $\Theta_{0}$ which is specified as the max. pith to CS border radius.

Polar-transformed CS-Images can be treated like polar iris images. Compared to the size of the iris, CSs are larger and the transformation is not restricted to an annular shaped ring. In case of more than 64 annual rings the common polar transformation format of $512 \times 64$ pixels causes a loss of information. Our results in [15] showed that the larger format significantly outperforms the smaller one. Consequently, for this work just the larger format with $512 \times 512$ pixels is utilized.

Two feature extractors from the USIT package [12] were extended to work with a larger polar CS-Image format of $512 \times 512$ pixels. First, the Log Gabor (LG) algorithm by Masek [9] was extended in accordance with the original algorithm by defining the region of interest (ROI) through a number of rows $r$ with a height $h_{r}$. Like the original, a row is condensed into a 1-D signal which is run through the Gabor filtering process. Since it is not clear which configuration of $r$ and $h_{r}$ is best we choose to use a variance of combinations, including combinations where the ROI does not span the whole polar-transformed CS-Image. However, unlike the iris biometry case which excludes the outer iris boundary, which frequently exhibits occlusions by cilia, we choose to exclude the inner residual part of the polar CS-Image. This part consists of a low number of pixels which are stretched to the polar CS-Image width, thus providing nearly no usable information. Note that the size of the feature vector is dependent on $h_{r}$.

Furthermore the algorithm by Ko et al. (KO) [7] was simply adopted by allowing bigger textures without adapting the cell-size which is averaged. Note that as a result the length of the feature vector increases with the size of the texture. The extended algorithms are directly applied to the polar-transformed and CLAHE enhanced CS-Images. Both algorithms compute a CS-Code composed of a single feature vector.

Iris-based matching procedures For the matching of irisbased CS-Codes KO uses a specific comparator [7] and LG the Hamming distance. Figure 5 shows a schematic overview of iris-based rotation compensation. If the rotational prealignment vector $\Theta_{0}$ is computed different for the same input CS-Image a given point in both images $\left(E_{1}\right)$ appears at different angular locations, the radial distance stays the same. The rotational variance is reduced to a translation in the normalized texture.

The feature vector values (=bits) of the iris-based CS-Code are extracted along a fixed grid in the normalized texture. Consequently, a bit shift in the iris-based CS-Code corresponds to a translation in the normalized texture. In this way bit-shifts on the iris-based CS-Codes are suited to compensate remaining rotational variations. 


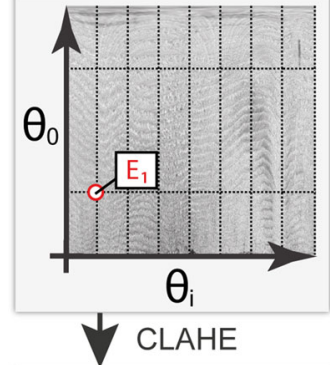

Feature Extraction

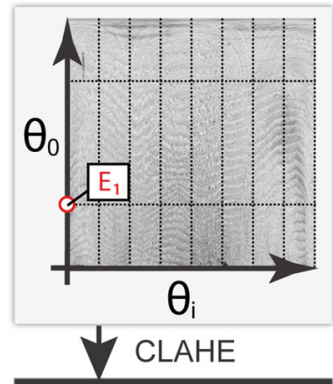

Feature Extraction

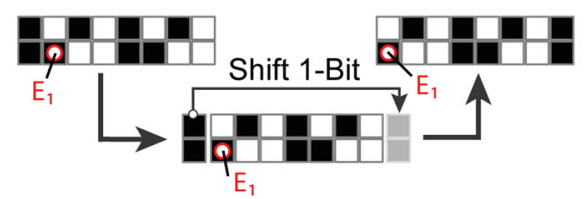

Fig. 5 Iris-based CS-Code Shifting

Similar as for the fingerprint-based matching procedure, the final MS between two iris-based CS-Codes is determined between all combinations of shifted CS-Codes.

\section{Experiments}

In the experimental evaluation verification and identification performances for different configurations are assessed. Additionally, the rotational pre-alignment performances are assessed using an image registration algorithm. Introductory, the testsets are outlined and the experimental setup for the utilized configurations is described.
Testsets $\left(\mathrm{TS}_{1}, \mathrm{TS}_{2}, \mathrm{TS}_{3}\right)$ The first two testsets were already used for the experiments in $[15,16]$. The CS-Images of TS 1 and $\mathrm{TS}_{2}$ were captured with a tripod and showed nearly no variances with respect to affine transformations. For $\mathrm{TS}_{1} 50$ different tree logs were captured four times with and without flash. Additionally, the ends of eight logs were cross-cut and captured once again, with and without flash. In $\mathrm{TS}_{2} 120$ $\operatorname{logs}$ were captured three times without flash (in $[15,16] 105$ of $\mathrm{TS}_{2}$ were used). The new testset $\mathrm{TS}_{3}$ consists of 109 different logs where each log was captured between 10 to 14 times without flash. All images were taken handholding the camera which was rotated for each new CS-Image. For each CS-Image the pith position and the CS border were determined manually and are utilized as groundtruth data for the experiments. Samples of each testset are shown in Fig. 6.

\subsection{Experimental set-up}

For all CS-Images of the testsets CS-Codes and MSs were computed for different configurations of the fingerprint- and iris-based approaches.

Fingerprint configurations Remaining rotational variances are compensated by computing feature vectors for rotations in the range from $-8^{\circ}$ to $8^{\circ}$ in $2^{\circ}$ steps. The CS-Codes are computed using $16 \times 16$ non-overlapping blocks. The Gabor filterbank is build up on six different filters tuned to eight directions.

Iris configurations Remaining rotational variances are compensated by shifting the iris-based CS-Codes in a range between -21 to 21 feature vector positions. This approximately equals the rotation compensation range of $-8^{\circ}$ to $8^{\circ}$ for the fingerprint configurations.
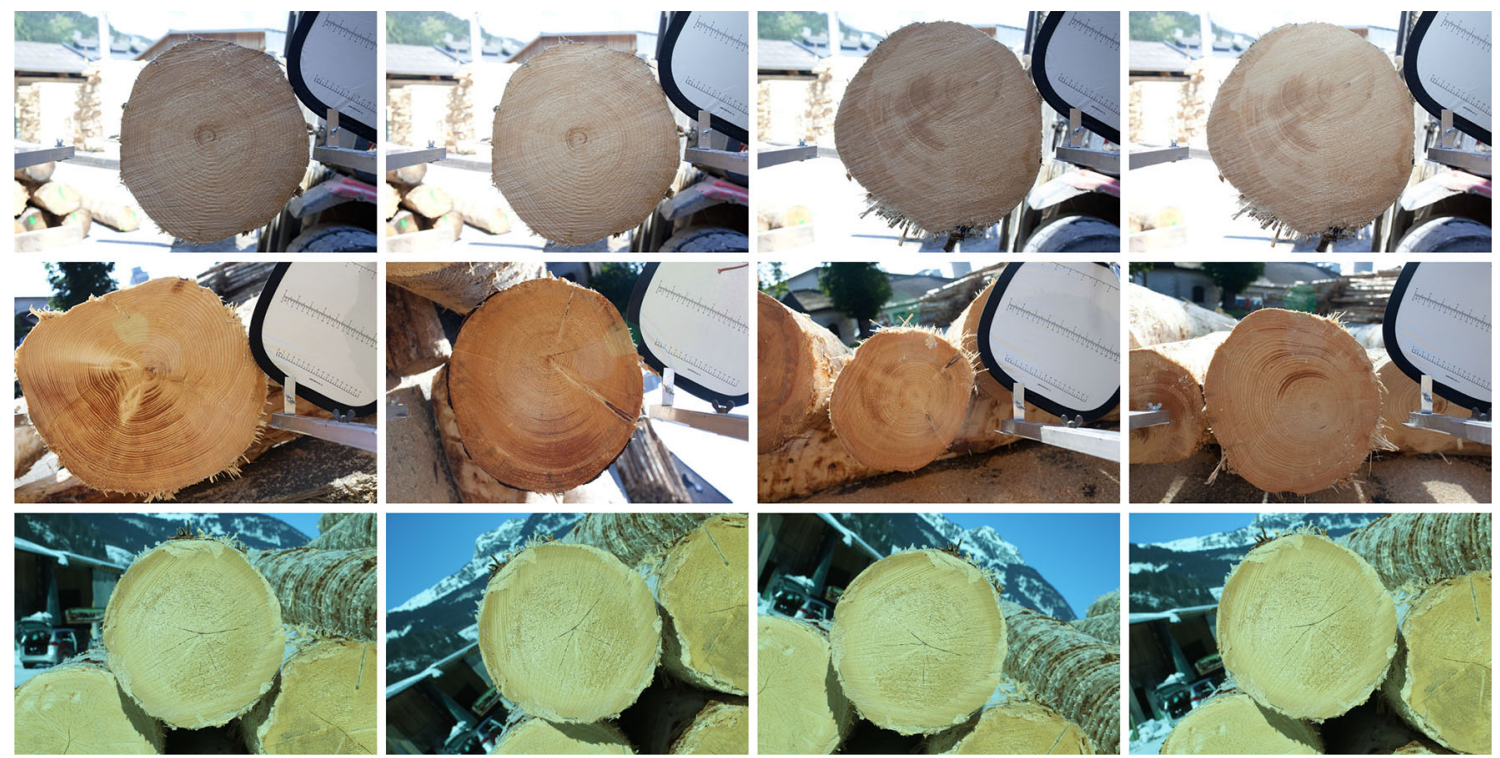

Fig. 6 1st Row $\left(\mathrm{TS}_{1}\right)$ : Respectively, two CS-Images from two different logs—one captured with flash and one without flash. 2 nd Row (TS 2 ): Four CS-Images from different logs. 3rd Row $\left(\mathrm{TS}_{3}\right)$ : Four CS-Images illustrating the rotational variations 
Furthermore, results for IRIS ${ }_{V}$ and IRIS ${ }_{H}$ are presented. IRIS $_{H}$ means that the annual ring patterns in the polartransformed CS-Image are aligned horizontally as common. For IRIS ${ }_{V}$ the polar-transformed CS-Image is rotated by $90^{\circ}$. Commonly, feature extraction in iris recognition aims to extract information from vertically aligned structures and thus may performs better if we rotate the polar-transformed CS-Images. Note, that in case of IRIS $V$ CS-Code shifting does not correspond to a rotational shift in the input CSImage. More or less, this corresponds to vertically shifting the unrolling center (=pith position) up/down in the input CS-Image. For LG three different configurations in terms of number of rows and row height LG $\left(r, h_{r}\right)$ are utilized: LG $(16,32)$, LG $(64,08)$ and LG $(50,10)$.

\subsection{Results and discussion}

The experimental evaluation is performed in three stages. First, the performances of the rotation compensation strategies are assessed utilizing image registration algorithms. Second, the verification performances for all fingerprint/iris configurations and all rotational pre-alignment strategies are assessed. Finally, we assess the identification performances of the best configurations in case of rotational pre-alignment.

For a better examination the results in each stage are provided for $\mathrm{TS}_{1}$ and $\mathrm{TS}_{2}$ together $\left(\mathrm{TS}_{12}\right), \mathrm{TS}_{3}$ and all three testsets (ALL). Additionally, all configurations are assessed without rotational pre-alignment (NORC).

\subsubsection{Rotational pre-alignment performance}

The performances of the rotational pre-alignment strategies are assessed using the image registration approach by [19] (provided for MATLAB) which gives an estimate for the rotational error between two images.

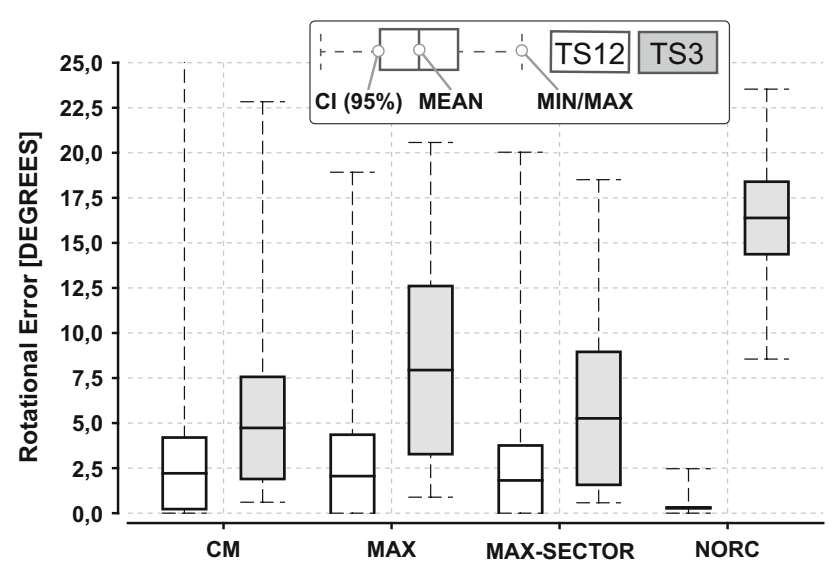

Fig. 7 Rotational variations computed by the image registration approach proposed in [19]
All CS-Image of $\mathrm{TS}_{12}, \mathrm{TS}_{3}$ were rotationally pre-aligned using the three different strategies. Subsequently, for each strategy the remaining rotational errors between the prealigned CS-Images of each log were computed employing accurate image registration [19]. The rotational error statistics for each strategy (mean, variance and confidence intervals for a level of $95 \%$ ) each strategy and $\mathrm{TS}_{12}, \mathrm{TS}_{3}$ are depicted in Fig. 7. Results for NORC confirm that the rotational variations in $\mathrm{TS}_{12}$ are very low and that $\mathrm{TS}_{3}$ shows large rotational variations. The rotational variations in $\mathrm{TS}_{3}$ cover a range of approximately $45^{\circ}$. Furthermore, the results indicate that all rotational pre-alignment strategies are suited to reduce rotational variations in $\mathrm{TS}_{3}$. Results for $\mathrm{TS}_{12}$ show that all rotational pre-alignment strategies introduce rotational variations. Taking all statistics into account the lowest rotational variances are shown for the MAX-SECTOR rotational pre-alignment strategy.

\subsubsection{Verification performance evaluation}

The verification performance is assessed based on the Equal Error Rate (EER) and the margin of error (MOE) achieved for each configuration. The MOE is estimated for a $95 \%$ level of confidence using subset partitioning [3]. The EERs computed for all configurations, rotational pre-alignment strategy and testsets are shown in Table 1. To improve the overview EERs for some configurations are summarized and the MOEs are only shown for the CM pre-alignment strategy. Note, that the results for NORC and $\mathrm{TS}_{12}$ are comparable to the results presented in [15].

Rotation compensation strategies Initially, the verification performances achieved for the different rotational pre-alignment strategies are assessed. In case of NORC the EERs for $\mathrm{TS}_{12}$ are much better than the EERs for $\mathrm{TS}_{3}$. This confirms the results of the rotational pre-alignment performance evaluation and shows that the large rotational variances in $\mathrm{TS}_{3}$ cannot be compensated by the template computation and matching procedures of the fingerprintand iris-based approaches. The impact of rotational prealignment becomes obvious comparing the EERs for $\mathrm{TS}_{3}$ achieved for NORC to those achieved with rotational prealignment (MAX, MAX-SECTOR and CM). The best (=lowest) EERs for $\mathrm{TS}_{3}$ and ALL are achieved when CM is used for pre-alignment. In case of rotational pre-alignment this also accounts for the $\mathrm{TS}_{12}$ EERs. Furthermore, the results show that for all configurations the EERs for MAX-SECTOR are better (=lower) than for MAX. Considering the EERs it can be stated that CM is the best of the three rotational prealignment strategies, especially when considering the results for $\mathrm{TS}_{3}$.

Fingerprint-based configurations In case of the fingerprint-based configurations the results for NORC-MS ${ }_{\mathrm{AP}}$ differ from the results presented in [15]. Compared to the EER 
Table 1 EERs $[\%]$ and \pm MOE $[\%]$ for the fingerprint- and iris-based configurations
Table 2 LG $(64,08)$-EERs[\%] for CS-Code shifting/no CS-Code shifting

\begin{tabular}{|c|c|c|c|c|c|c|c|c|c|c|c|c|c|}
\hline \multirow{2}{*}{\multicolumn{2}{|c|}{ Configuration }} & \multicolumn{3}{|c|}{ NORC } & \multicolumn{3}{|c|}{ MAX } & \multicolumn{3}{|c|}{ MAX-SECTOR } & \multicolumn{3}{|c|}{$\mathrm{CM}$} \\
\hline & & $5^{2}$ & $\Sigma^{\infty}$ & $\underset{\nabla}{~}$ & $\Sigma^{N^{\prime \prime}}$ & $\Sigma^{\infty}$ & 국 & $\hat{S}^{2}$ & $\Sigma^{\infty}$ & $\vec{\nabla}$ & $\delta^{2}$ & $\hat{N}^{\infty}$ & $\underset{\nabla}{\checkmark}$ \\
\hline \multirow{3}{*}{ 诖 } & $\mathrm{MS}_{A P}$ & 1.22 & \multirow{3}{*}{$\hat{\wedge}$} & \multirow{3}{*}{$n$} & \multirow{3}{*}{$\begin{array}{l}0 \\
\wedge\end{array}$} & \multirow{3}{*}{$\stackrel{+}{\wedge}$} & \multirow{3}{*}{$\stackrel{\wedge}{\wedge}$} & \multirow{3}{*}{$\stackrel{m}{\wedge}$} & \multirow{3}{*}{$\stackrel{m}{\wedge}$} & \multirow{3}{*}{$\stackrel{n}{\wedge}$} & $1.11 \pm 0.5$ & $1.28 \pm 2.9$ & $1.26 \pm 0.5$ \\
\hline & $\mathrm{MS}_{F}$ & 0.35 & & & & & & & & & $1.54 \pm 1.3$ & $6.85 \pm 2.3$ & $5.54 \pm 0.8$ \\
\hline & $\mathrm{MS}_{A P, F}$ & 0.54 & & & & & & & & & $0.64 \pm 0.4$ & $1.32 \pm 2.1$ & $1.41 \pm 0.5$ \\
\hline \multirow{4}{*}{$\begin{array}{l}\mathbb{\pi} \\
\cong \\
\cong\end{array}$} & $\mathrm{LG}(16,32)$ & 0.4 & \multirow{4}{*}{ 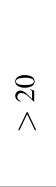 } & \multirow{4}{*}{$\stackrel{ }{\wedge}$} & \multirow{4}{*}{$\stackrel{+}{\wedge}$} & \multirow{4}{*}{$\frac{\sim}{\wedge}$} & \multirow{4}{*}{$\vec{\wedge}$} & \multirow{4}{*}{$\stackrel{n}{\wedge}$} & \multirow{4}{*}{$\begin{array}{l}0 \\
\wedge\end{array}$} & \multirow{4}{*}{$n$} & $1.32 \pm 1.1$ & $2.44 \pm 0.4$ & $2.06 \pm 0.8$ \\
\hline & $\mathrm{LG}(64,08)$ & 0.97 & & & & & & & & & $2.13 \pm 1.3$ & $2.29 \pm 0.5$ & $2.12 \pm 0.7$ \\
\hline & $\operatorname{LG}(50,10)$ & 0.67 & & & & & & & & & $1.92 \pm 0.9$ & $2.39 \pm 0.5$ & $2.55 \pm 0.9$ \\
\hline & $\mathrm{KO}$ & 0.99 & & & & & & & & & $5.28 \pm 1.5$ & $7.89 \pm 1.8$ & $7.33 \pm 0.9$ \\
\hline \multirow{4}{*}{$\begin{array}{l}\vec{n} \\
\frac{2}{\Xi}\end{array}$} & $\operatorname{LG}(16,32)$ & 0.92 & \multirow{4}{*}{$\begin{array}{l}0 \\
\wedge\end{array}$} & \multirow{4}{*}{$\stackrel{n}{\wedge}$} & \multirow{4}{*}{$\stackrel{m}{\wedge}$} & \multirow{4}{*}{$\hat{\wedge}$} & \multirow{4}{*}{$\hat{\wedge}$} & \multirow{4}{*}{$\stackrel{\wedge}{\wedge}$} & \multirow{4}{*}{$\stackrel{+}{\wedge}$} & \multirow{4}{*}{$\stackrel{+}{\wedge}$} & $0.91 \pm 0.6$ & $1.21 \pm 1.7$ & $1.26 \pm 0.5$ \\
\hline & $\mathrm{LG}(64,08)$ & 0.83 & & & & & & & & & $1.11 \pm 0.8$ & $0.81 \pm 1.8$ & $0.9 \pm 0.6$ \\
\hline & $\mathrm{LG}(50,10)$ & 0.67 & & & & & & & & & $0.97 \pm 0.9$ & $1.14 \pm 1.7$ & $0.9 \pm 0.6$ \\
\hline & $\mathrm{KO}$ & 0.96 & & & & & & & & & $3.15 \pm 1.1$ & $3.81 \pm 1.9$ & $3.48 \pm 0.7$ \\
\hline
\end{tabular}

$E E R<2 \%$ : Yellow coloured results signalize all EERs $<2 \%$. Best TS EER \& MOE: Green coloured and bold face marked results show the best EERs and MOEs for each testset achieved with rotational pre-alignment

\begin{tabular}{lllll}
\hline Configuration & NORC & MAX & MAX-SECTOR & CM \\
\hline IRIS $_{H}$ & $20.51 / 50.80$ & $12.11 / 28.21$ & $6.67 / 17.83$ & $2.12 / 18.68$ \\
\hline IRIS $_{V}$ & $5.46 / 6.74$ & $7.18 / 7.81$ & $3.79 / 3.86$ & $0.90 / 0.96$ \\
\hline
\end{tabular}

achieved for $\mathrm{MS}_{\mathrm{AP}}-\mathrm{ENH}_{\mathrm{NO}}(15.7 \%)$ in [15] the EER for NORC-MS $\mathrm{AP}_{\mathrm{AP}}-\mathrm{TS}_{12}(1.22 \%)$ is much better. This improvement is attributed to the better-contrast normalization due to CLAHE. In our previous works we applied local contrast normalization and the size of the local regions was probably set too large. However, the $\mathrm{MS}_{\mathrm{AP}}-\mathrm{ENH}_{1}$ and $\mathrm{ENH}_{2}$ results in [15] (1.7 and $0.9 \%)$ are in the range of the results for NORC-MS ${ }_{\mathrm{AP}}-\mathrm{TS}_{12}$ in this work $(1.22 \%)$.

The results for $\mathrm{MS}_{F}$ lead to new interesting insights. In case of NORC-MS $-\mathrm{TS}_{12}(0.35 \%)$ the shape has a stronger discriminative power than the annual ring pattern $\left(\mathrm{MS}_{\mathrm{AP}}=\right.$ $1.22 \%)$. Fusion of both leads to no further improvement of the EER $\left(\mathrm{MS}_{\mathrm{AP}, F}=0.54 \%\right)$. In case of CM feature fusion fusion improves the EER and MOE for $\mathrm{TS}_{12}$ ( $\mathrm{MS}_{\mathrm{AP}, F}=0.64 \pm 0.4 \%$ ). The best fingerprint-based results for $\mathrm{TS}_{3}$ and ALL are achieved with CM-MS $\mathrm{AP}$ and account 1.28 and $1.26 \%$, respectively. Fusion leads to no improvement.

Iris-based configurations For the iris-based configurations the EERs for all LG configurations and NORC - TS 12 are slightly worse compared to the ENH NO results shown in [15]. Note that testset TS 12 was extended by CS-Images of $15 \mathrm{logs}$ and we use a different contrast normalization in this work. On the other hand, it seems that KO profits from CLAHE and the EER for KO-NORC-TS $12(0.99 \%)$ is much better than the comparable EER for KO-ENH $\mathrm{NO}_{\mathrm{NO}}(2.73 \%)$ in [15].
IRIS $_{H}$ vs. IRIS $S_{V}$ Basically, for most of the iris configurations the IRIS $V$ results outperform the IRIS $_{H}$ results. As noted, iris recognition aims to extract vertical aligned structures. All results for IRIS $V$ clearly confirm that the utilized feature extractors perform better if we rotate the polar-transformed CS-Images. The overall best EER with $0.9 \pm 0.6 \%$ for ALL testsets in Table 1 is achieved with IRIS $_{V}$-CM-LG $(50,10)$ and LG $(64,08)$.

IRIS $_{H} /$ IRIS $_{V}$ CS-Code shifting The improvements for IRIS $_{V}$ are astonishing because CS-Code shifting in the matching procedure is not suited to compensate rotational variations. To illustrate this, in Table 2 we present EERs for LG $(64,08)$, IRIS $_{H}$ and IRIS $V$ where no CS-Code shifting in the matching procedure is performed.

For a better overview the EERs achieved with shifting are also shown. In case of IRIS $_{H}$ the results show that with shifting the EERs get better for each rotational pre-alignment strategy. On the other hand the results for IRIS $V$ demonstrate that the results are not that improved by shifting. We assume that shifting in case of IRIS $_{V}$ is suited to compensate small radial variations. However, accompanied by the results in Table 2 the results confirm the assumption that $\mathrm{KO}$ and $\mathrm{LG}$ achieve a significantly better performance in case of textures showing vertically aligned structures.

Basic insights In accordance with our results in [15] the verification performance evaluation shows that the EERs for the best fingerprint- and iris-based configurations are quite 


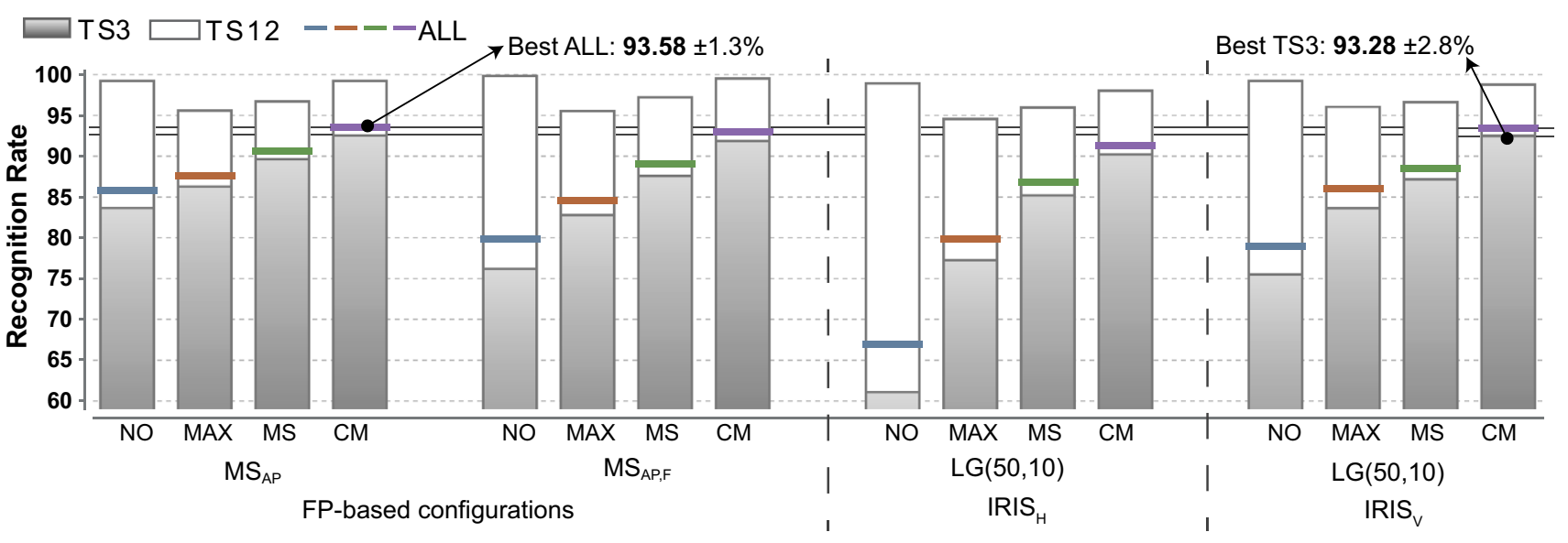

Fig. 8 Identification performance evaluation—Rank 1 detection rates

low and show a high degree of separability between the intra- and interclass score distributions for a large set of tree logs. Considering the rotational variations in the new $\mathrm{TS}_{3}$ results show that rotational pre-alignment in the registration procedure improves the robustness to rotational variations. Furthermore, the results for $\mathrm{TS}_{12}$ in case of NORC and $\mathrm{CM}$ indicate that there is nearly no decrease in the verification performance due to rotational pre-alignment. Note, that in case of rotational pre-alignment rotational variations are introduced to $\mathrm{TS}_{12}$ which basically contains no rotational variations.

\subsection{Identification performance evaluation}

For the identification performance evaluation, the best configurations for the fingerprint-based IRIS $_{H}$ and IRIS $_{V}$ configurations were preselected, and the respective results are depicted for all rotational pre-alignment strategies in Fig. 8. For each configuration and the different testsets, the Rank 1 identification rates are presented.

In accordance with the verification performance results, the recognition rates for $\mathrm{TS}_{12}$ are better than for $\mathrm{TS}_{3}$. The total recognition rate for ALL is somewhere in-between. For the fingerprint-based configurations the results for $\mathrm{TS}_{12}$ and $\mathrm{MS}_{\mathrm{AP}, F}$ show that the fusion of annual ring pattern and shape information does increase the identification performance significantly. In case of $\mathrm{TS}_{3}$ and ALL the results for $\mathrm{MS}_{\mathrm{AP}, F}$ show that fusion decreases the identification performances slightly.

For IRIS $_{H}$ and IRIS ${ }_{V}$ the best configurations with respect to the best recognitions rates for ALL are shown. The IRIS ${ }_{H}$ and IRIS $_{V}-\mathrm{LG}(50,10)$ configurations confirm that for $\operatorname{IRIS}_{V}$ the identification performance increases significantly. The best identification rate and $\mathrm{MOE}(93.58 \pm 1.3 \%)$ for ALL is achieved with $\mathrm{MS}_{\mathrm{AP}}$ and CM. In difference to the verification performance results the identification rates for $\mathrm{CM}$ are not that excessively better than those achieved with the other rotational pre-alignment strategies. However, same as in the verification performance evaluation results show that for all configurations the CM rotational pre-alignment strategy achieves the highest identification rates, especially when considering the results for $\mathrm{TS}_{3}$ and ALL.

\section{Conclusions}

This work demonstrates that rotational pre-alignment in the registration procedure is suited to overcome rotational variations in log end biometrics. Along with our results in [15] it is shown that fingerprint and iris recognition techniques can be successfully transferred to the field of wood log tracking. Based on the variety of 279 different logs the results indicate the applicability of $\log$ end biometrics to log identification.

Due to the best verification performance results and the high identification rates, we conclude that $\mathrm{CM}$ is the best of the investigated rotational pre-alignment strategies. For the fingerprint-based approach the results show that the annual ring pattern feature $\mathrm{MS}_{\mathrm{AP}}$ - ALL achieves a very good EER for CM (1.26\%). No improvement by including shape information in the matching procedure $\left(\mathrm{MS}_{\mathrm{AP}, F}\right)$ can be observed. In case of the iris-based approaches LG outperforms the results of $\mathrm{KO}$ and IRIS $V$ performs better than IRIS $_{H}$. The best EER $(0.9 \pm 0.6 \%)$ is achieved with IRIS $_{V}-\mathrm{CM}$ $\mathrm{LG}(50,10)$ or $\mathrm{LG}(64,08)$. In the identification performance experiments the best fingerprint and iris-based configurations achieve detection rates $>93 \%$ at Rank 1 .

The next stage of our research will be to investigate the impact of automated pith estimation [17] and CS segmentation [18] on the biometric system performance. Furthermore, the performance for IRIS $_{V}$ raises the general question for the best filters and filter parameters and to assess further feature descriptors for feature extraction. 
Acknowledgements Open access funding provided by [Paris Lodron University of Salzburg].

Open Access This article is distributed under the terms of the Creative Commons Attribution 4.0 International License (http://creativecomm ons.org/licenses/by/4.0/), which permits unrestricted use, distribution, and reproduction in any medium, provided you give appropriate credit to the original author(s) and the source, provide a link to the Creative Commons license, and indicate if changes were made.

\section{References}

1. Barrett, W.: Biometrics of cut tree faces. In: Sobh, T. (ed.) Advances in Computer and Information Sciences and Engineering, pp. 562565. Springer, Netherlands (2008)

2. Berglund, A., Broman, O., Grönlund, A., Fredriksson, M.: Improved log rotation using information from a computed tomography scanner. Comput. Electron. Agric. 90, 152-158 (2013)

3. Bolle, R.M., Ratha, N.K., Pankanti, S.: Error analysis of pattern recognition systemsthe subsets bootstrap. Comput. Vis. Image Underst. 93(1), 1-33 (2004)

4. Jain, A., Ross, A., Prabhakar, S.: Fingerprint matching using minutiae and texture features. In: Proceedings of the International Conference on Image Processing (ICIP'01), vol. 3, pp. 282-285. Thessaloniki, GR (2001)

5. Jain, A.K., Ross, A.A., Nandakumar, K.: Introduction to Biometrics. Springer, New York (2011)

6. Karel, Z.: Graphics Gems iv. Chap. Contrast Limited Adaptive Histogram Equalization. Academic Press Professional, Inc., San Diego (1994)

7. Ko, J.G., Gil, Y.H., Yoo, J.H., Chung, K.I.: A novel and efficient feature extraction method for iris recognition. ETRI J. 29(3), 399_ 401 (2007)

8. Maltoni, D., Maio, D., Jain, A.K., Prabhakar, S.: Handbook of Fingerprint Recognition. Springer, New York (2009)

9. Masek, L.: Recognition of human iris patterns for biometric identification. Master's thesis, University of Western Australia (2003)

10. Norell, K., Borgefors, G.: Estimation of pith position in untreated $\log$ ends in sawmill environments. Comput. Electron. Agric. 63(2), 155-167 (2008)

11. Ramoser, H., Wachmann, B., Bischof, H.: Efficient alignment of fingerprint images. In: Proceedings of the 16th International Conference on Pattern Recognition, vol. 3, pp. 748-751 (2002)

12. Rathgeb, C., Uhl, A., Wild, P.: Iris Recognition: From Segmentation to Template Security, Advances in Information Security, vol. 59. Springer (2013)

13. Schraml, R., Charwat-Pessler, J., Petutschnigg, A., Uhl, A.: Towards the applicability of biometric wood log traceability using digital log end images. Comput. Electron. Agric. 119, 112-122 (2015)

14. Schraml, R., Charwat-Pessler, J., Uhl, A.: Temporal and longitudinal variances in wood log cross-section image analysis. In: IEEE International Conference on Image Processing (ICIP'14). Paris, FR (2014)
15. Schraml, R., Hofbauer, H., Petutschnigg, A., Uhl, A.: Tree log identification based on digital cross-section images of log ends using fingerprint and iris recognition methods. In: Proceedings of the 16th International Conference on Computer Analysis of Images and Patterns (CAIP'15), LNCS, pp. 752-765. Springer (2015)

16. Schraml, R., Petutschnigg, A., Uhl, A.: Validation and reliability of the discriminative power of geometric wood log end features. In: Proceedings of the IEEE International Conference on Image Processing (ICIP'15) (2015)

17. Schraml, R., Uhl, A.: Pith estimation on rough log end images using local fourier spectrum analysis. In: Proceedings of the 14th Conference on Computer Graphics and Imaging (CGIM'13). Innsbruck, AUT (2013)

18. Schraml, R., Uhl, A.: Similarity based cross-section segmentation in rough log end images. In: Iliadis, L., et al. (eds.) Proceedings of the 10th Artificial Intelligence Applications and Innovations Conference (AIAI'14), IFIP AICT, vol. 436, pp. 614-621. Springer, Rhodes, GR (2014)

19. Vandewalle, P., Ssstrunk, S., Vetterli, M.: A frequency domain approach to registration of aliased images with application to superresolution. EURASIP J. Appl. Signal Process. (special issue on Super-resolution) 2006 (2006). Source Code: http://lcav.epfl.ch/ software/superresolution

Alexander Petutschnigg is a full professor and head of department for Forest Products Technology at the University of Applied Sciences Salzburg. His research interests are in supply chain optimization, product- and process development, wood and biogenic resources.

Rudolf Schraml is a PhD student at the University of Salzburg. His research interests are in wood image processing focusing on biometric tracking of wood logs using digital log end images.

Andreas Uhl is a full professor at the Computer Sciences Department at the University of Salzburg, Austria, where he leads the Multimedia Signal Processing and Security Lab. His research interests are in image and video processing, biometrics, visual data encryption, medical image analysis, and high-performance computing.

Heinz Hofbauer holds a doctoral degree in computer science. He works as a researcher at the University of Salzburg and has published extensively in the fields of content and media security and biometrics. 